\title{
Stretch-induced changes in constricted lung parenchymal strips: role of extracellular matrix
}

\author{
F.G. Salerno*, A. Fust\#, M.S. Ludwig ${ }^{\#}$
}

Stretch-induced changes in constricted lung parenchymal strips: role of extracellular matrix. F. G. Salerno, A. Fust, M.S. Ludwig. (C) ERS Journals Ltd 2004.

ABSTRACT: Large amplitude oscillations of contracted airway smooth muscle cause relative relaxation of the preparation. However, little is known about the effect of mechanical stretch on distal lung behaviour.

Rat parenchymal strips were suspended in an organ bath and attached at one end to a force transducer and at the other end to a servo-controlled lever arm that effected length changes. Mechanical impedance of the strip was measured by applying a complex signal consisting of pseudorandom length oscillations of varying frequencies $(0.5-19.75 \mathrm{~Hz})$. A constant phase model was fit to changes in length and tension to calculate tissue damping (G) and elastance (H). Hysteresivity was calculated as $\mathbf{G} / \mathbf{H}$. Impedance was measured before and after sinusoidal length oscillation at different amplitudes $(1,3,10$ and $25 \%$ of resting length) at a frequency of $1 \mathrm{~Hz}$ under baseline conditions and after acetylcholine-induced constriction.

Oscillations of 10 and $25 \%$ amplitudes significantly decreased the $\mathbf{G}$ and $\mathbf{H}$ of the lung strip. The effect of length oscillations was no different in control versus constricted strips.

These data suggest that in the distal lung, large stretches affect the structural components of the extracellular matrix rather than the contractile elements.

Eur Respir J 2004; 23: 193-198.
* Respiratory Unit, Salvatore Maugeri Foundation, Research Institute, Cassano Murge (BA), Italy and "Meakins-Christie Laboratories, McGill University, Montreal, QC, Canada.

Correspondence: M.S. Ludwig, MeakinsChristie Laboratories, McGill University, 3626 St Urbain Street, Montreal, QC, Canada $\mathrm{H} 2 \mathrm{X} 2 \mathrm{P} 2$

Fax: 15143987483

E-mail: mara.ludwig@mcgill.ca

Keywords: Complex impedance contractile elements

tissue mechanics

Received: March 62003

Accepted after revision: September 152003

This work was supported by the J.T. Costello Memorial Research Fund and the Canadian Institutes of Health Research.
Understanding the mechanisms underlying the effect of a deep inspiration on lung mechanics has implications for the understanding of asthma pathophysiology. Deep inspirations taken before or after a methacholine challenge decrease the bronchoconstrictor response in normal subjects but not in asthmatics [1, 2]. Normal subjects, when asked to refrain from taking deep inspirations after a methacholine (MCh) challenge, behave similarly to hyperresponsive subjects [3]. These observations suggest that the protective effect of deep inspirations on airway narrowing is reduced or absent in asthmatics, a characteristic that may differentiate them from normal subjects. Studies in isolated airway smooth muscle (ASM), which attempt to reproduce the in vivo airway response to tidal breathing and deep inspirations, show that ASM constricts less under dynamic conditions [4]. These data suggest that the effect of lung volume fluctuations on airway narrowing comes from alterations in the ASM.

An alternative hypothesis is that the differential effect of deep inspiration in asthmatics compared with controls relates to different lung tissue mechanical properties, because of differences in the extracellular matrix or because of effects on airway-parenchymal interdependence. The lung tissue is a viscoelastic material, and, by definition, viscoelastic materials display hysteresis. FroEb and MEAD [5] theorised that airways also display hysteresis, and the relative hysteresis of these two structures would determine the effect of a deep breath on airway calibre because of the mechanical interdependence between airways and the surrounding lung parenchyma $[6,7]$. If the hysteretic properties of asthmatic lungs are different compared with normal lungs, deep inspirations could result in bronchoconstriction [8].

The energy dissipation that occurs during volume oscillation of the lung is attributable not only to viscoelastic but also to plastic behaviour [9]. Deep stretches may affect airway calibre because of amplitude-dependent plastic deformation of the lung tissue or airway wall. Plastic deformation of these structures could result in altered elastic and hysteretic properties, with the final effect of an altered load on the ASM.

While the effect of deep inspirations on large airways, and of stretch on isolated ASM has been well investigated in both humans and animals $[2,4,10]$, relatively little is known about the effect of stretch on peripheral lung contraction. It was reasoned that acetylcholine (Ach)-induced constriction of the peripheral lung would be reversed by large amplitude stretch, similar to what occurs in the airways. Rat lung parenchymal strips were utilised to investigate this question. Lung parenchymal strips have been used as a model for the study of lung peripheral mechanical and pharmacological behaviour [11-15]. Rats represent an appropriate model, as extensive studies have been published using this species as an animal model of asthma [16, 17]. Studies in control animals permit investigation of this question before the more complex situation of asthmatic disease is addressed. Therefore, rat parenchymal strips were stretched, both under baseline conditions and after induced constriction, and oscillationinduced modifications in the mechanics compared. 


\section{Methods}

\section{Tissue preparation}

Sprague Dawley rats weighing $\sim 350 \mathrm{~g}$ were studied. Each animal was anaesthetised with a peritoneal injection of sodium pentobarbital $\left(30 \mathrm{mg} \cdot \mathrm{kg}^{-1}\right)$. After tracheostomy, a metal cannula (internal diameter of $2 \mathrm{~mm}$ ) was inserted into the trachea and tightly bound. Through an abdominal incision, the diaphragm was cut and a bilateral pneumothorax was induced. The thorax was opened, the animal exsanguinated and the heart, lungs and trachea were carefully resected en bloc. The lungs were filled and rinsed to total lung capacity with a modified Krebs solution at a $\mathrm{pH}$ of 7.40 and a temperature of $6^{\circ} \mathrm{C}$. Lung parenchymal strips $(3 \times 3 \times 10 \mathrm{~mm})$ were cut, the pleura was dissected, and unloaded length (Lo) and wet weight of each strip recorded.

\section{Apparatus}

Metal clips were glued to either end of the tissue strip with cyanoacrylate. Steel music wires $(0.5 \mathrm{~mm}$ diameter $)$ were attached to the clips and the strip suspended vertically in an organ bath. A mercury bead was placed in the bottom of the organ bath to prevent the Krebs solution from leaking out. The bath was filled with $15 \mathrm{~mL}$ of Krebs solution, maintained at $37^{\circ} \mathrm{C}$ and continuously bubbled with $95 \% \mathrm{O}_{2} /$ $5 \% \mathrm{CO}_{2}$. One end of the strip was attached to a force transducer (model 400A; Cambridge Technologies, Watertown, MA, USA) while the other end was connected to a servo-controlled lever arm (model 300B; Cambridge Technologies). The lever arm was in turn connected to a function generator (model 3030; B \& K Precision, Dynascan Corporation, Chicago, IL, USA), which controlled the frequency, amplitude and waveform of the oscillation. Movement of a screw-thumb wheel system, which effected vertical displacements of the force transducer, set the resting tension. Length and force signals were converted from analogue to digital (DT2801-A; Data Translation Inc., Marlborough, MA, USA) low-pass filtered, and recorded on computer at a sampling frequency of $256 \mathrm{~Hz}$. For impedance measurements, an 8-s broad-band pseudorandom displacement signal composed of 17 mutually primed frequencies ranging $0.5-19.75 \mathrm{~Hz}$ and an
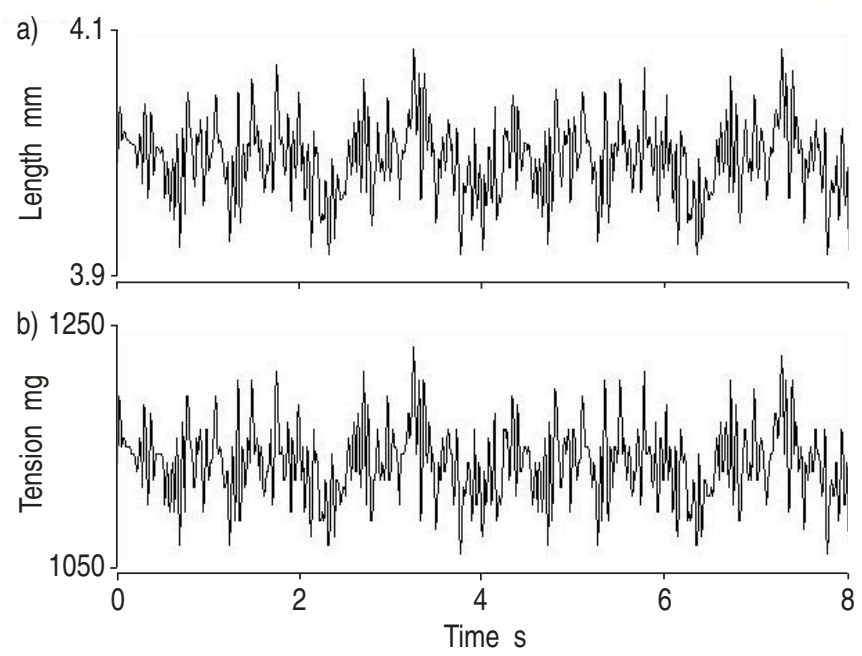

Fig. 1. - Length and tension signals during the pseudorandom length perturbation. amplitude up to $0.18 \mathrm{~mm}$ was computer-generated and delivered to the lever arm (fig. 1).

\section{Protocol}

A total of six strips from six animals were studied ( $\mathrm{n}=6$ for amplitude $(\epsilon)=10$ and $25 \% ; n=5$ for $\epsilon=1$ and $3 \%$ ). Each lung parenchymal strip was preconditioned by slowly cycling tension from 0 to $2 \mathrm{~g}$ three times; on the third cycle the strip was unloaded to $\sim 1.2 \mathrm{~g}$ and allowed to stabilise for $45 \mathrm{~min}$ at the end of which time the tension was $\sim 1 \mathrm{~g}$. Complex impedence was measured using the pseudorandom displacement signal under baseline conditions and after 5 min of sinusoidal length oscillations above the mean operating tension at an amplitude of either 1, 3, 10 or $25 \%$ of Lo and a frequency of $1.0 \mathrm{~Hz}$. After oscillation, tension was reset to the same baseline value, a 30 -min stabilisation period was allowed, and a new baseline was recorded. Thereafter, strips were challenged with ACh of $0.1 \mathrm{mM}$ (Sigma, Oakville, ON, Canada) for 5 min until a stable level of constriction was obtained. Complex impedance measurements were obtained before and after $5 \mathrm{~min}$ of oscillations at the same amplitude as before. In addition to the complex impedance measurements, tension was measured continuously for the duration of the sinusoidal oscillations, as well as during the ACh challenge (fig. 2). ACh was then washed from the organ bath, and the preparation was allowed to restabilise for $45 \mathrm{~min}$. The protocol was then repeated for the remaining three amplitudes in random order.

\section{Data analysis}

Tissue impedance was calculated as:

$$
\mathrm{Z}=\mathrm{T} / \Delta \mathrm{L} / \Delta t
$$

where $\mathrm{Z}$ is mechanical impedance, $\mathrm{T}$ is tension, $\mathrm{L}$ is length and $t$ is time.

Tension and length signals were obtained and recorded as a function of time, Fourier transformed to functions of frequency, and complex impedance calculated. The mechanical parameters were estimated by fitting the constant phase model [18] to the impedance data, according to the formula:

$$
\mathrm{Z}=\mathrm{Rn}+\mathrm{j} \omega \mathrm{In}+\frac{(\mathrm{G}-\mathrm{jH})}{\omega^{\alpha}}
$$

where $\mathrm{Rn}$ is Newtonian resistance, $\mathrm{G}$ is tissue damping, $\mathrm{H}$ is

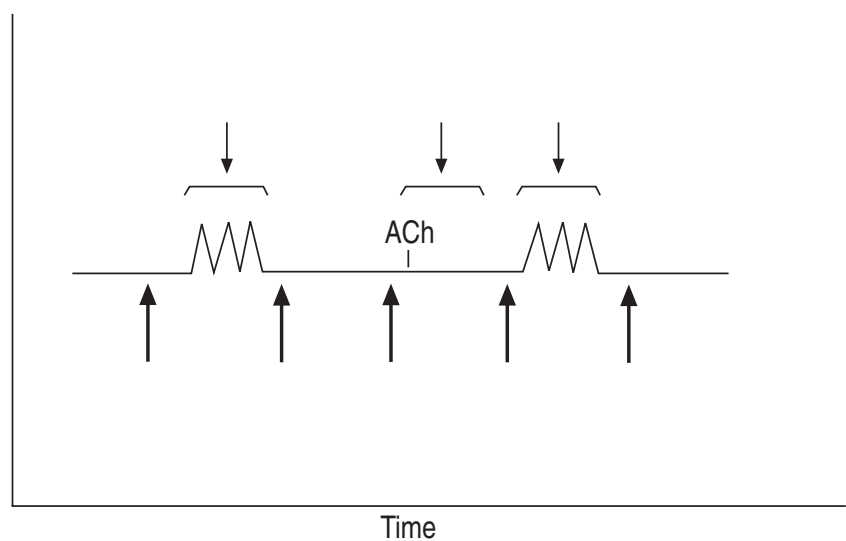

Fig. 2.-Experimental protocol. Thick arrows represent impedance measurements and the thin arrows represent continuous recording of tension. Ach: acetylcholine. 
tissue elastance, In is inertance, $\omega$ is angular frequency, and $\mathrm{j}$ is an imaginary unit (fig. 3). Results were standardised for strip size by multiplying the values of $\mathrm{G}$ and $\mathrm{H}$ by Lo/Ao. Ao $\left(\mathrm{cm}^{2}\right)$ is the unstressed cross-sectional area of the strip obtained from the formula:

$$
\mathrm{A}_{\mathrm{o}}=\frac{\mathrm{W}_{\mathrm{o}}}{\rho \times \mathrm{L}_{\mathrm{o}}}
$$

where Wo is the wet weight in $g$, $\rho$ is the mass density of the tissue taken as $1.06 \mathrm{~g} \cdot \mathrm{cm}^{-3}$ and Lo is the unloaded length in $\mathrm{cm}$.

Hysteresivity, $\eta$, a dimensionless variable coupling dissipative and elastic behaviour, was calculated as:

$$
\eta=\frac{\mathrm{G}}{\mathrm{H}}
$$

t-tests were used to assess whether oscillations had an effect on the mechanical parameters, and to compare the effect of oscillations in the unconstricted versus the constricted state. Analysis of variance (ANOVA; two-way) was used to determine the effect of amplitude on tension in the two conditions. Results were considered statistically significant at a probability level of $5 \%$. Values are reported as mean \pm SE.

\section{Results}

Baseline mechanics are shown in table 1. There were no differences in baseline mechanics among the four amplitudes. The per cent change in all mechanical parameters after induced constriction is shown in table 2. ACh, during all amplitudes, caused a significant increase in tension, tissue damping and tissue elastance, except tissue damping during the $3 \%$ amplitude.

The effect of oscillation amplitude on tension, tissue damping and tissue elastance and hysteresivity before and after induced constriction is shown in figure 4. Oscillations caused a significant decrease in tension except at $3 \%$ amplitude in the unconstricted state (fig. 4a). Tissue elastance significantly decreased in the unconstricted state at 10 and $25 \%$ amplitude and in the constricted state at 3, 10 and $25 \%$ amplitude (fig. 4b). Tissue damping significantly decreased at 1 and $25 \%$ amplitude in the constricted state and at 10 and $25 \%$ amplitude in the unconstricted state (fig. 4c). Hysteresivity was affected by 1 and $25 \%$ length oscillations in the constricted state (fig. 4d).

When the effect of oscillation on mechanics was compared

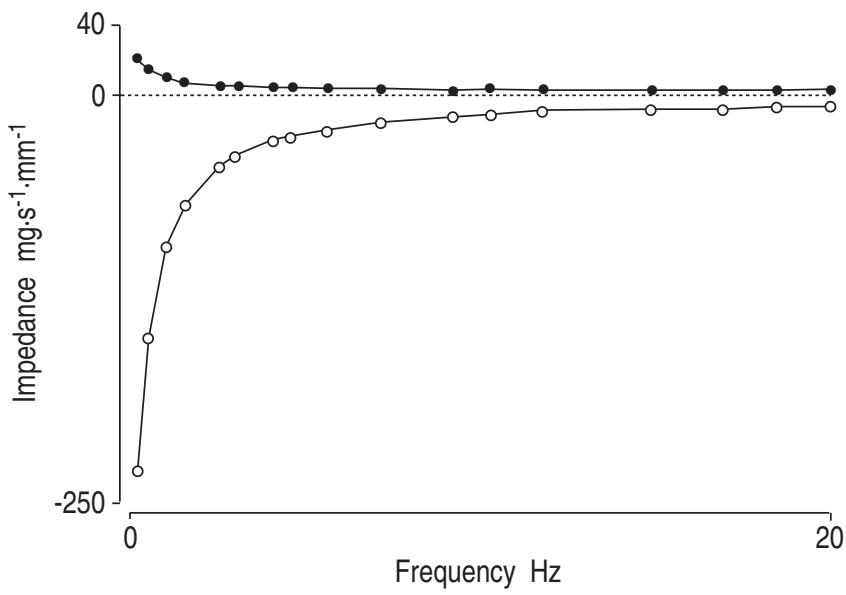

Fig. 3.-Complex impedance versus frequency data for a typical recording. : tissue damping; $\bigcirc$ : tissue elastance.
Table 1.-Baseline mechanics of the parenchymal strips

\begin{tabular}{lcccc}
\hline & \multicolumn{4}{c}{ Amplitude \% } \\
\cline { 2 - 5 } & 1 & 3 & 10 & 25 \\
\hline $\mathrm{T} \mathrm{mg}$ & $1073 \pm 31$ & $1088 \pm 41$ & $1080 \pm 39$ & $1059 \pm 29$ \\
$\mathrm{H} \mathrm{mg} \cdot \mathrm{mm}^{-2}$ & $606 \pm 46$ & $602 \pm 33$ & $598 \pm 36$ & $631 \pm 44$ \\
$\mathrm{G} \mathrm{mg} \cdot \mathrm{mm}^{-2}$ & $60.4 \pm 4.0$ & $59.8 \pm 4.2$ & $67.2 \pm 7.8$ & $61.1 \pm 6.6$ \\
$\eta$ & $0.101 \pm 0.005$ & $0.100 \pm 0.006$ & $0.112 \pm 0.009$ & $0.096 \pm 0.006$
\end{tabular}

Data are presented as mean \pm SEM. T: tension; H: elastance; G: tissue damping; $\eta$ : hysteresivity.

in the unconstricted versus the constricted state, generally no significant differences were detected (fig. 4). A modest difference in tension at 1 and $3 \%$ amplitude $(\mathrm{p}<0.05)$, and in tissue elastance at $1 \%$ amplitude $(\mathrm{p}<0.05)$ was observed. These differences were minimal and unlikely to be of physiological significance.

The absolute changes in tension during the oscillation as a function of amplitude are shown in figure 5. A dose/response relationship between amplitude and decrease in tension was observed; oscillation amplitude had a significant effect on the decrease in tension (two-way ANOVA; $\mathrm{p}<0.001$ ). At all amplitudes there were no differences in the decrease in tension between the unconstricted versus the constricted state.

\section{Discussion}

The main observation of this study was that large stretches significantly affect tension, tissue damping and elastance of the lung parenchymal strip; this effect was essentially independent of whether the contractile apparatus was activated. These findings suggest that large amplitude stretch affects the structural components of the extracellular matrix of the peripheral lung rather than reversing induced constriction of the contractile elements.

It is well established that lung volume history has a profound effect on lung mechanics. Deep breaths in normal subjects cause a transient modification in airway calibre after an MCh challenge [2]. In addition, when normal subjects avoid deep inspirations during an $\mathrm{MCh}$ challenge, airway hyperresponsiveness develops [3]. The same phenomenon has been demonstrated in animals; GuNST et al. [19] showed that, in rabbits, deep inspirations imposed during or after an $\mathrm{MCh}$ challenge decrease the maximum constrictor response. Similarly, tidal ventilation significantly affected airway responsiveness to $\mathrm{MCh}$ in an amplitude-dependent fashion; larger tidal volume fluctuations render the lung less responsive to agonist challenge [20,21].

In isolated, constricted, large ASM, length oscillations induce a decrement in force and stiffness in an amplitudeand frequency-dependent fashion, reproducing the volume

\begin{tabular}{|c|c|c|c|c|}
\hline & \multicolumn{4}{|c|}{ Amplitude $\%$} \\
\hline & 1 & 3 & 10 & 25 \\
\hline $\mathrm{T} \%$ & $6.3 \pm 0.8^{*}$ & $6.4 \pm 1.6^{*}$ & $6.0 \pm 1.3^{*}$ & $5.9 \pm 0.7^{*}$ \\
\hline $\mathrm{H} \%$ & $20.2 \pm 2.6^{*}$ & $21.2 \pm 7.5^{*}$ & $22.0 \pm 3.5^{*}$ & $21.8 \pm 1.9^{*}$ \\
\hline $\mathrm{G} \%$ & $14.0 \pm 5.1^{*}$ & $10.3 \pm 13.2$ & $31.5 \pm 7.7 *$ & $21.9 \pm 6.2 *$ \\
\hline$\eta \%$ & $-4.6 \pm 5.3$ & $-6.1 \pm 13.6$ & $7.5 \pm 4.4$ & $0.0 \pm 5.5$ \\
\hline
\end{tabular}

Table 2.-Per cent increase after acetylcholine challenge

Data are presented as mean \pm SEM. T: tension; H: elastance; $\mathrm{G}$ : tissue damping; $\eta$ : hysteresivity. ${ }^{*}: \mathrm{p}<0.05$ versus baseline. 

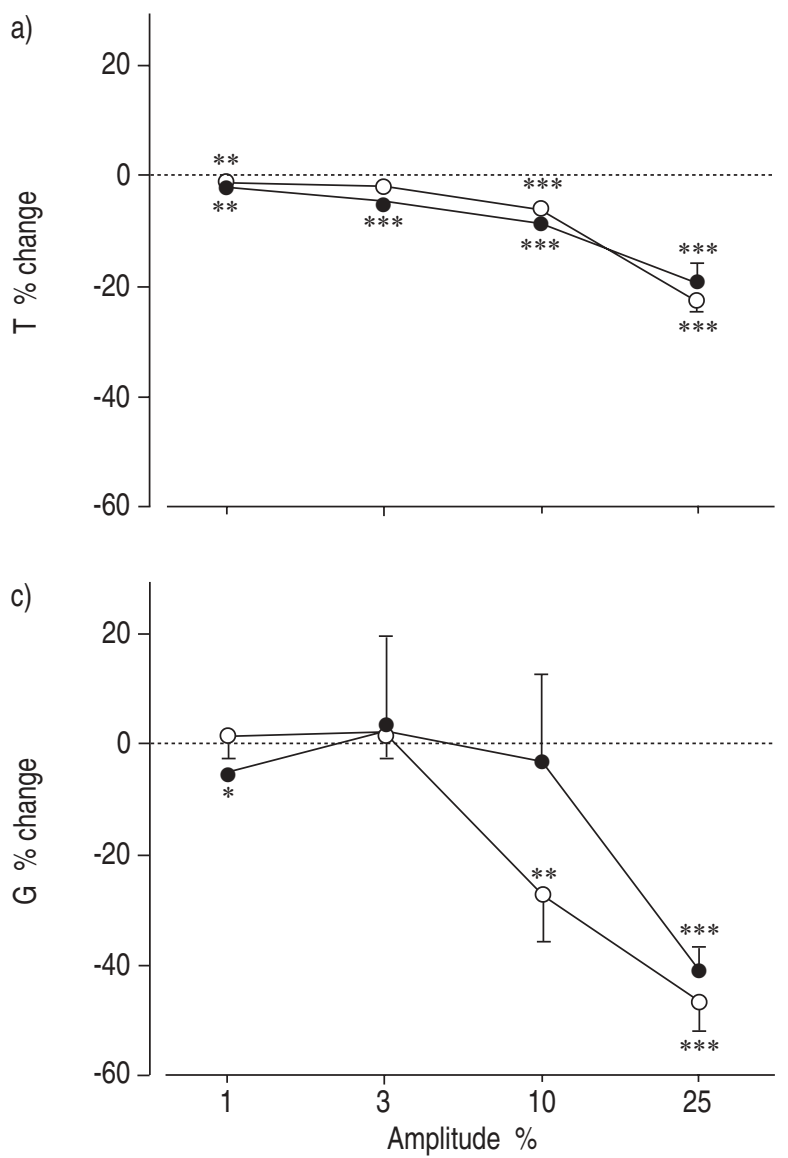
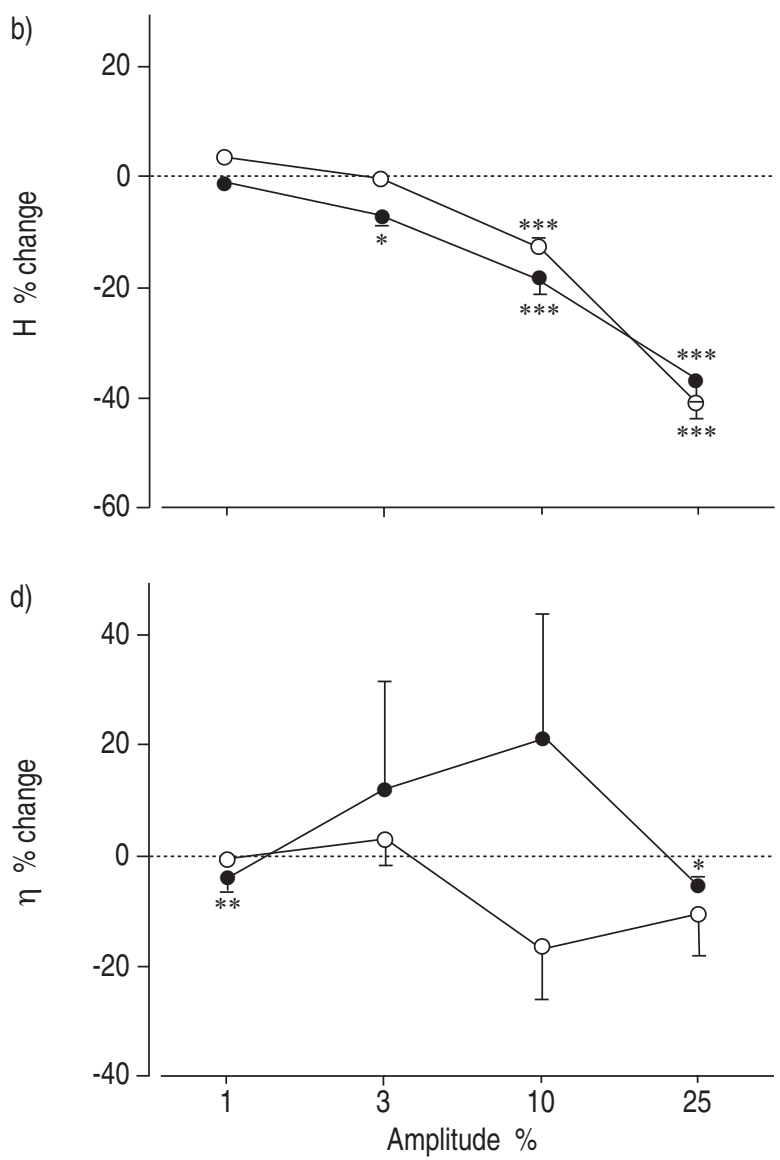

Fig. 4. - Percentage change from baseline in a) tension (T), b) elastance $(\mathrm{H})$, c) tissue damping $(\mathrm{G})$, and d) hysteresivity $(\eta)$ in the constricted ( and unconstricted $(\bigcirc)$ state, after oscillation at four different amplitudes. $*$ : $\mathrm{p}<0.05$ versus baseline; $* *$ : $\mathrm{p}<0.01$ versus baseline; $* * *$ : $\mathrm{p}<0.001$ versus baseline.

effect seen in vivo [4]. GuNST et al. [22] have proposed that reorganisation of the contractile apparatus caused by stretch is responsible for the reduced ASM response seen under dynamic conditions. Pratusevich et al. [23] have suggested that the number of contractile units in series depend on the adapted muscle length. Muscle length fluctuations would reduce force because filaments are moved from their optimal overlap. WANG et al. [10] have recently reported that unstimulated tracheal smooth muscle also shows a change in tension with length conditioning, again implicating plasticity of the cellular organisation in determining the mechanical characteristics of the smooth muscle. Finally, FREDBERG et al. [24] have suggested that during ASM length cycling, the changes in contractility are largely accounted for by the rupture of cross bridges which have formed between the myosin heads and the actin filaments.

There is relatively little in the literature on the effect of lung volume fluctuations on mechanical behaviour of the distal lung. The authors questioned whether stretch would affect induced constriction of the lung periphery. Modification of the parenchymal tissue may affect the load that the smooth muscle in larger airways must overcome during bronchoconstriction. Owing to interdependence, changes in parenchymal mechanics would affect this load, both under baseline conditions, and even more so, during bronchoconstriction when the parenchyma, especially that around the airways, undergoes local stretch and distortion. Theoretically, the difference in the effect of deep inspiration on airway calibre in the normal versus the asthmatic population, could be determined by a different effect of lung volume change on the mechanics of the constricted lung tissue.

The distal lung is a complex system comprised of alveolar ducts, alveolar walls, small airways and small vessels. The lung parenchymal strip is considered a good proxy of the peripheral lung tissue, since it contains all of these elements

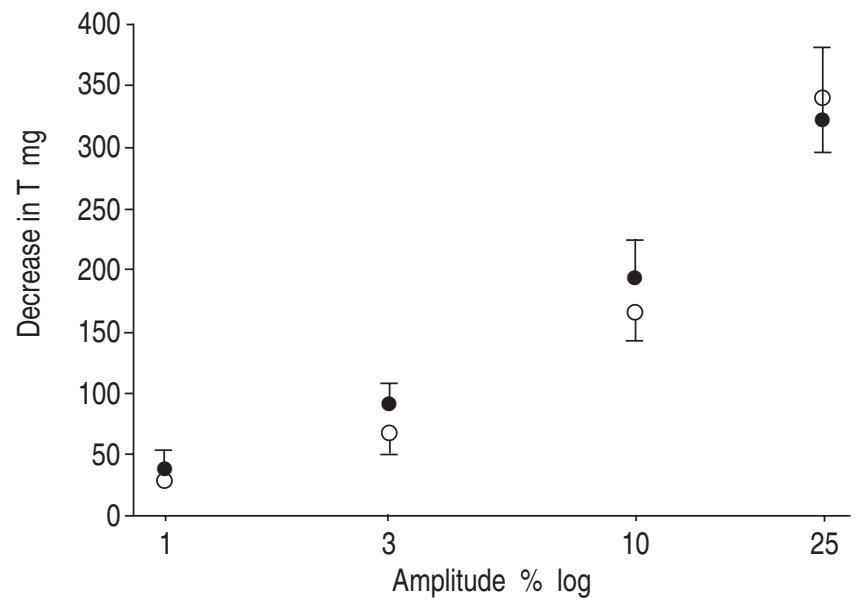

Fig. 5.-Decrease in tension (T) versus oscillation amplitude (log scale), in the constricted $(\bullet)$ and unconstricted $(\bigcirc)$ state. There was no difference in the effect of the oscillation amplitude on tension between the two states (analysis of variance). 
and is a commonly used preparation in the study of the mechanical and pharmacological properties of the distal lung [11-15]. There are some limitations, however, to this approach. With the lung parenchymal strip, stretch is not three-dimensional, as occurs in vivo, but rather uniaxial, so that only the component of contraction occurring along one axis would contribute to the change in mechanics. Nonetheless, this approach has been used by a number of investigators who have proposed that an oscillation of $1-3 \%$ of resting length approximately reproduces the stretch that occurs during tidal volume ventilation; oscillations in the order of $25 \%$ resting length are thought to be in the range of deep inspiration [25]. This was the basis on which the authors chose the size of the oscillation, although the exact degree of volume change these oscillations reproduce is difficult to ascertain.

In this study, baseline mechanics were comparable with those previously reported $[15,26,27]$. When the lung parenchymal strip was subjected to a large mechanical strain $(10 \%$ and especially $25 \%$ amplitude), significant modifications in tension, tissue damping and elastance occurred. Hysteresivity was relatively unaffected. YUAN et al. [15] have shown, in guinea-pig lung parenchymal strips, a similar amplitude dependence of tissue damping and elastance, and lack of effect on $\eta$. Conversely, NAvAJAs et al. [28] did not find an appreciable effect of stretch amplitude on tissue elastance in dog lung parenchymal strips. In this experiment, the duration of the oscillations was relatively short and the strip size was considerably larger. In addition, the oscillations were performed around the mean operating stress, whereas in the current experiment oscillations were performed on top of the mean operating stress. Stretches on top of the operating stress more closely mimic in vivo deep inspirations, and result in a higher mean stress for a given amplitude of oscillation.

Lung parenchyma is a viscoplastoelastic material. The amplitude dependence of tissue damping and elastance is consistent with plasticity or nonlinear viscoelasticity [9]. Different anatomic mechanisms have been proposed to account for this behaviour. The extracellular matrix, the surface film and the contractile apparatus, all may contribute to the viscoplastoelastic properties of the lung. The extracellular matrix consists of collagen and elastic fibres, glycoproteins and proteoglycans. MijaILOVICH et al. [29] suggested that fibre-fibre interactions are the main source of the viscoplastic behaviour. SUKI et al. [30] proposed that "reptation" of the collagen and elastic fibres inside a solution of biopolymers accounts for the viscoelastic properties of the lung tissue. Fibres in the lung, as well as in other tissues, are embedded in a matrix consisting of many different molecules, including proteoglycans. Proteoglycans are hydrophilic molecules that modulate tissue turgor and thereby, mechanical properties. Al JAMAL et al. [31] have recently shown that exposure to enzymes that degrade matrix glycosaminoglycans, alter the viscoelastic properties of the lung parenchymal strip. These data suggest that the energy dissipation at the tissue level is attributable to the ground substance, in addition to the collagen and elastic fibre network.

Tissue damping, tissue elastance and tension increased when the strips were challenged with ACh. This response was comparable with that obtained in previous work from the authors' laboratory, as well as that of others [14, 32]. When lung parenchymal strips are challenged with smooth muscle agonists, a constrictor response is observed. Different contractile elements in the lung periphery may be responsible for the contractile response. Small airways, small blood vessels, as well as interstitial contractile cells [33] may all contribute to the observed changes in peripheral mechanics induced by ACh. It is not established, however, to what extent the changes in the mechanics reflect the response of the smooth muscle present in the preparation, or contractioninduced modification of other elements in series or parallel with the smooth muscle, such as the collagen-elastinproteoglycan matrix [34].

Constricted lung strips showed an amplitude-dependent decrease in tension, tissue damping and elastance, similar to that obtained in the unconstricted state. It may be expected that length oscillations would cause a decrease in the mechanical parameters equivalent to the sum of the mechanical change during baseline plus reversal in the Ach-induced activation of the contractile apparatus. However, the changes seen in the mechanics were not significantly different in the two experimental conditions. These data suggest that large mechanical stretches, while altering the mechanics of the ultrastructural components of the matrix, do not reverse the changes in mechanics directly caused by the activation of the contractile machinery. YUAN et al. [15] measured complex impedance at various strain amplitudes in guinea-pig parenchymal strips in which the tissues were rendered nonviable. There were no differences in the mechanics or in the effects of strain amplitude regardless of the viability of the smooth muscle within the preparation. They concluded that the connective tissue matrix dominates parenchymal mechanics. However, in this experiment, the parenchymal preparation was not actively constricted during length oscillations. The present results are also in accord with data recently published by WANG et al. [10]. These authors showed that, in tracheal smooth muscle, length-induced shifts in the passive and active length/tension curves were equivalent. These findings implicate changes in the "extracellular scaffolding" and/or intracellular cytoskeleton distinct from the contractile element. The present data are the first to show that, in the distal lung, amplitude effects are also related to the structural components of the preparation, rather than activated smooth muscle per se.

There are potential implications of the findings for airway hyperresponsiveness. Large lung volume swings, by altering parenchymal mechanics, will impact on the load airway smooth muscle must overcome in order to shorten. A further consideration is the mechanism of relative hysteresis. A change in the hysteretic properties of the parenchyma relative to the airways may explain bronchoconstriction or bronchodilation following a deep breath. Hence, in asthmatics, differences in the response of the lung periphery to large volume oscillations may contribute to differences in the airway response to volume oscillation. Remodelling of the airways is well described in asthma [35]. Resultant changes in the viscoelastic properties of the airway wall could alter the airway response to a deep inspiration. Whether the lung parenchyma undergoes similar remodelling requires investigation, but several investigators have demonstrated inflammation at the level of the distal lung [36, 37]. Hence, it seems likely that alteration in the mechanical behaviour of the parenchyma and the impact of a deep breath on the distal lung contribute to asthma pathophysiology.

\section{References}

1. Kapsali T, Permutt S, Laube B, Scichilone N, Togias A. Potent bronchoprotective effect of deep inspiration and its absence in asthma. J Appl Physiol 2000; 89: 711-720.

2. Nadel JA, Tierney DF. Effect of a previous deep inspiration on airway resistance in man. J Appl Physiol 1961; 16: 717719.

3. Skloot G, Permutt S, Togias A. Airway hyperresponsiveness in asthma: a problem of limited smooth muscle relaxation with inspiration. J Clin Invest 1995; 96: 2393-2403.

4. Gunst SJ. Contractile force of canine airway smooth muscle 
during cyclical length changes. J Appl Physiol 1983; 55: 759769.

5. Froeb HF, Mead J. Relative hysteresis of the dead space and lung in vivo. J Appl Physiol 1968; 25: 244-248.

6. Macklem PT. Mechanical factors determining maximum bronchoconstriction. Eur Respir J 1989; 2: Suppl. 6, 516s519s.

7. Mead J, Takishima T, Leith D. Stress distribution in lungs: a model of pulmonary elasticity. J Appl Physiol 1970; 28: 596608.

8. Burns CB, Taylor WR, Ingram RH Jr. Effects of deep inhalation in asthma: relative airway and parenchymal hysteresis. J Appl Physiol 1985; 59: 1590-1596.

9. Stamenovic D, Glass GM, Barnas GM, Fredberg JJ. Viscoplasticity of respiratory tissues. J Appl Physiol 1990; 69: 973-988.

10. Wang L, Parè PD, Seow CY. Selected contribution: effect of chronic passive length change on airway smooth muscle length-tension relationship. J Appl Physiol 2001; 90: 734-740.

11. Dolhnikoff M, Morin J, Ludwig MS. Human lung parenchyma responds to contractile stimulation. Am J Respir Crit Care Med 1998; 158: 1607-1612.

12. Goldie RG, Bertram JF, Papadimitriou JM, Paterson JW. The lung parenchyma strip. Trends Pharmacol Sci 1984; 5: 7-9.

13. Lulich KM, Mitchell HW, Sparrow MP. The cat lung strip as an in vitro preparation of peripheral airways: a comparison of beta-adrenoreceptor agonists, autacoids and anaphylactic challenge on the lung strip and trachea. Br J Pharmacol 1976; 56: 71-79.

14. Salerno FG, Dallaire M, Ludwig MS. Does the anatomic makeup of parenchymal lung strips affect oscillatory mechanic during induced constriction? J Appl Physiol 1995; 79: 66-72.

15. Yuan H, Ingenito EP, Suki B. Dynamic properties of lung parenchyma: mechanical contributions of fiber network and interstitial cells. J Appl Physiol 1997; 83: 1420-1431.

16. Bellofiore S, Martin JG. Antigen challenge of sensitized rats increases airway responsiveness to methacholine. $J \mathrm{Appl}$ Physiol 1988; 65: 1642-1646.

17. Palmans E, Kips JC, Pauwels RA. Prolonged allergen exposure induces structural airway changes in sensitized rats. Am J Respir Crit Care Med 2000; 161: 627-635.

18. Hantos Z, Daroczy B, Csendes T, Suki B, Nagy S. Modeling of low-frequency pulmonary impedance in dogs. $J$ Appl Physiol 1990; 68: 849-860.

19. Gunst SJ, Shen X, Ramchandani R, Tepper RS. Bronchoprotective and bronchodilatory effects of deep inspiration in rabbits subjected to bronchial challenge. $J$ Appl Physiol 2001; 91: 2511-2516.

20. Salerno FG, Shinozuka N, Fredberg JJ, Ludwig MS. Tidal volume amplitude affects the degree of induced bronchoconstriction in dogs. $J$ Appl Physiol 1999; 87: 1674-1677.
21. Shen X, Gunst SJ, Tepper RS. Effect of tidal volume and frequency on airways responsiveness in mechanically ventilated rabbits. J Appl Physiol 1997; 83: 1202-1208.

22. Gunst SJ, Meiss RA, Wu MF, Rowe M. Mechanisms for the mechanical plasticity of tracheal smooth muscle. $\mathrm{Am}$ J Physiol 1995; 268: C1267-C1276.

23. Pratusevich VR, Seow CY, Ford LE. Plasticity in canine airway smooth muscle. J Gen Physiol 1995; 105: 73-94.

24. Fredberg JJ, Jones KA, Nathan M, et al. Friction in airway smooth muscle: mechanism, latch and implications in asthma. J Appl Physiol 1996; 81: 2703-2712.

25. Fredberg JJ, Inouye D, Miller B, et al. Airway smooth muscle, tidal stretches, and dynamically determined contractile states. Am J Respir Crit Care Med 1997; 156: 1752-1759.

26. Sakai H, Ingenito EP, Mora R, et al. Hysteresivity of the lung and tissue strip in the normal rat: effects of heterogeneities. J Appl Physiol 2001; 91: 737-747.

27. Yuan H, Kononov S, Cavalcante FS, Lutchen KR, Ingenito EP, Suki B. Effects of collagenase and elastase on the mechanical properties of lung tissue strips. J Appl Physiol 2000; 89: 3-14.

28. Navajas D, Maksym GN, Bates JH. Dynamic viscoelastic nonlinearity of lung parenchymal tissue. J Appl Physiol 1995; 79: $348-356$.

29. Mijailovich SM, Stamenovic D, Brown R, Leith DE, Fredberg JJ. Dynamic moduli of rabbit lung tissue and pigeon ligamentum propatagiale undergoing uniaxial cyclic loading. J Appl Physiol 1994; 76: 773-782.

30. Suki B, Barabasi AL, Lutchen KR. Lung tissue viscoelasticity: a mathematical framework and its molecular basis. J Appl Physiol 1994; 76: 2749-2759.

31. Al Jamal R, Roughley PJ, Ludwig MS. Effect of glycosaminoglycan degradation on lung tissue viscoelasticity. $\mathrm{Am}$ J Physiol 2001; 80: L306-L315.

32. Fredberg JJ, Bunk D, Ingenito E, Shore SA. Tissue resistance and the contractile state of lung parenchyma. J Appl Physiol 1993; 74: 1387-1397.

33. Kapanci Y, Assimacopoulos A, Irle C, Zwahlen A, Gabbiani G. "Contractile interstitial cells" in pulmonary alveolar septa: a possible regulator of ventilation/perfusion ratio? J Cell Biol 1974; 160: 375-392.

34. Romero PV, Zin WA, Lopez-Aguilar J. Frequency characteristics of lung tissue strip during passive stretch and induced pneumoconstriction. J Appl Physiol 2001; 91: 882-890.

35. Jeffrey PK. Remodeling in asthma and COPD. Am J Respir Crit Care Med 2001; 164: 828-838.

36. Kraft M, Djukamenovic R, Wilson S, Holgate ST, Martin RJ. Alveolar tissue inflammation in asthma. Am J Respir Crit Care Med 1996; 154: 1505-1510.

37. Minshall EM, Hogg JC, Hamid QA. Cytokine mRNA expression in asthma is not restricted to large airways. J Allergy Clin Inflamm 1998; 101: 386-390. 